\title{
Serial automated quantitative CT analysis in idiopathic pulmonary fibrosis: functional correlations and comparison with changes in visual CT scores
}

Joseph Jacob, 1,2,3凶

Phone 00447511033666

Email joseph.jacob@nhs.net

Brian J. Bartholmai, 1,2

Srinivasan Rajagopalan, 1,2

Maria Kokosi, 4

Ryoko Egashira, 5

Anne Laure Brun, 6

Arjun Nair, 7

Simon L. F. Walsh, 8

Ronald Karwoski, 9

Athol U. Wells, 4

1 Department of Radiology, Royal Brompton Hospital, Royal Brompton and Harefield NHS Foundation Trust, London, UK AQ1 AQ2

2 Division of Radiology, Mayo Clinic Rochester, Rochester, MN, USA

310 Wolsey Road, Northwood, Middlesex, HA6 2HW UK

4 Interstitial Lung Disease Unit, Royal Brompton Hospital, Royal Brompton and Harefield NHS Foundation Trust, London, UK

5 Department of Radiology, Saga Daigaku, City, Saga, Japan

6 Department of Radiology, Whittington Hospital, London, UK 
7 Department of Radiology, Guys and St Thomas' NHS Foundation Trust, London, UK

8 Department of Radiology, Kings College Hospital NHS Foundation Trust, London, UK

9 Department of Physiology and Biomedical Engineering, Mayo Clinic Rochester, Rochester, Minnesota, USA

\section{Abstract}

\section{Objectives}

To determine whether computer-based CT quantitation of change can improve on visual change quantification of parenchymal features in IPF.

\section{Methods}

Sixty-six IPF patients with serial CT imaging (6-24 months apart) had CT features scored visually and with a computer software tool: ground glass opacity, reticulation and honeycombing (all three variables summed as interstitial lung disease extent [ILD]) and emphysema. Pulmonary vessel volume (PVV) was estimated by computer only. Relationships between changes in $\mathrm{CT}$ features and forced vital capacity (FVC) were examined using univariate and multivariate linear regression analyses.

\section{Results}

On univariate analysis, changes in computer variables demonstrated stronger linkages to $\mathrm{FVC}$ change than changes in visual scores (CALIPER ILD: $\mathrm{R}^{2}=0.53, \mathrm{p}<0.0001$; Visual ILD: $\left.\mathrm{R}^{2}=0.16, \mathrm{p}=0.001\right)$. PVV increase correlated most strongly with relative FVC change $\left(\mathrm{R}^{2}=0.57\right)$. When PVV constituents (vessel size and location) were examined, an increase in middle zone vessels linked most strongly to $\mathrm{FVC}$ decline $\left(\mathrm{R}^{2}=0.57\right)$ and was independent of baseline disease severity (characterised by $\mathrm{CT}$ fibrosis extent, FVC, or DLco).

\section{Conclusions}


An increase in PVV, specifically an increase in middle zone lung vessels, was the strongest CT determinant of FVC decline in IPF and was independent of baseline disease severity.

\section{Key Points}

- Computer analysis improves on visual CT scoring in evaluating deterioration on $C T$

- Increasing pulmonary vessel volume is the strongest CT predictor of functional deterioration

- Increasing pulmonary vessel volume predicts functional decline independent of baseline disease severity

\section{Keywords}

Multidetector-row computed tomography

Pulmonary fibrosis, idiopathic

Computer-assisted image Analysis

Idiopathic interstitial pneumonias

Blood vessels

\section{Electronic supplementary material}

The online version of this article ( https://doi.org/10.1007/s00330-017-5053-z ) contains supplementary material, which is available to authorized users.

\section{Introduction}

The current accepted gold standard for identifying clinical deterioration in patients with IPF is the measurement of forced vital capacity (FVC) decline [1]. However recent advances in volumetric CT analysis using advanced computer algorithms [2] appear to suggest that computer-based quantification of change in CT parenchymal patterns could similarly identify deterioration in IPF patients.

Only three studies to date have evaluated serial CT imaging in IPF and all have been constrained by small patient numbers or non-standardised CT acquisition parameters $[3,4,5]$. In the current study we aimed to explore the relationships between changes in various CT parameters, scored semi-quantitatively using visual analysis and quantitatively using computer analysis, with changes in key pulmonary function indices in a cohort of IPF patients. 


\section{Materials and methods}

\section{Study population}

The study population consisted of all new patients with a multidisciplinary team diagnosis of IPF according to published guidelines [1], presenting to the Royal Brompton Hospital over a four and a half year period (January 2007 to July 2011). Patients that had undergone two non-contrast, supine, volumetric thin section CT scans within a 6-24-month time period were selected. Exclusions are shown in the flowchart in Fig. 1. Standardised pulmonary function protocols were utilised $[2,6]$.

Fig. 1

Flowchart illustrating the selection of patients for the final study population

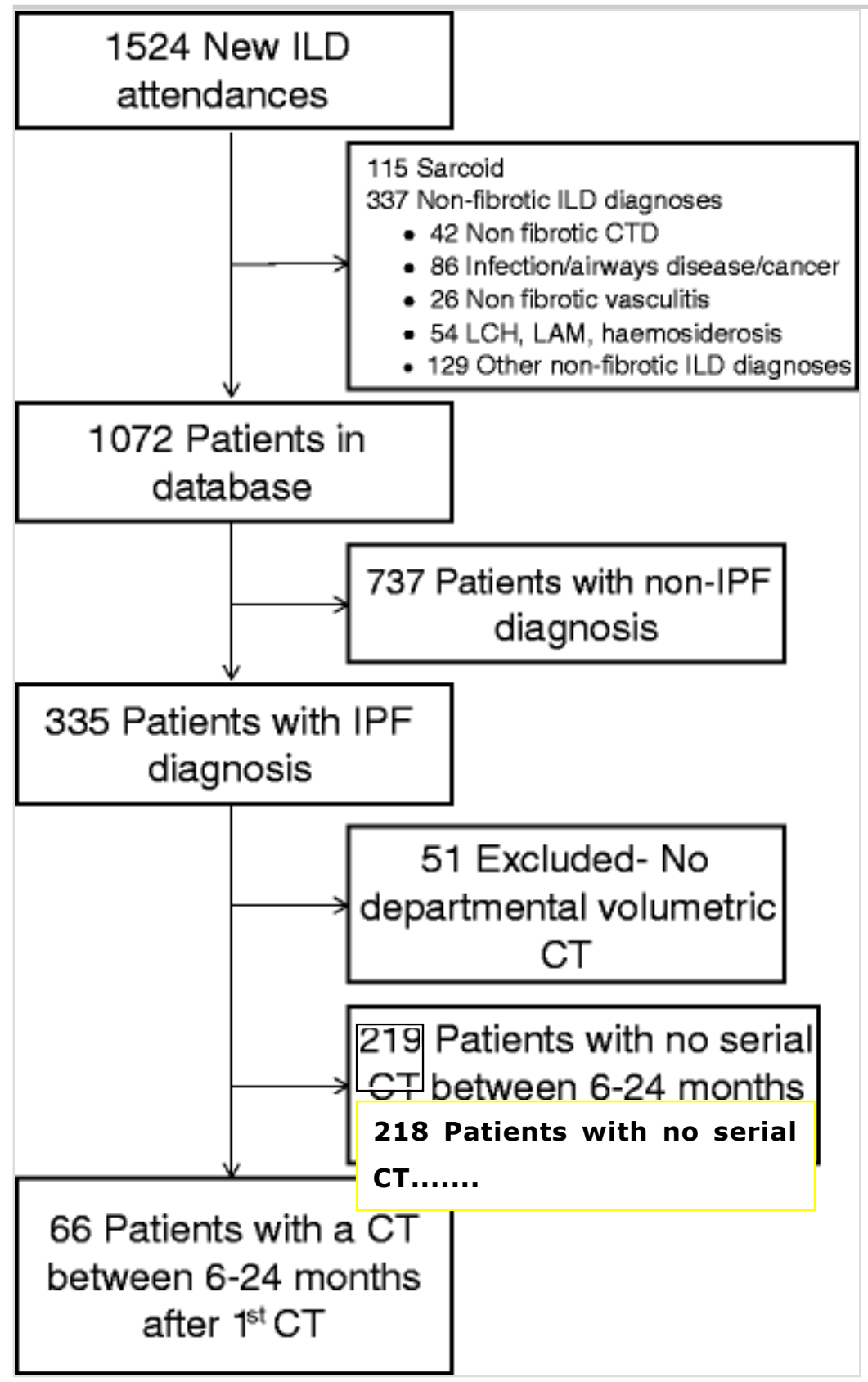




\section{CT protocol}

The CT scans were obtained using a 64-slice multiple detector CT scanner (Somatom Sensation 64, Siemens, Erlangen, Germany) or a 4-slice multiple detector CT scanner (Siemens Volume Zoom, Siemens, Erlangen, Germany). All scans were reconstructed using a high spatial frequency, B70 kernel (Siemens, Munich, Germany). All patients were scanned from lung apices to bases, supine, at full inspiration, with $1.0 \mathrm{~mm}$ section thicknesses using a peak voltage of $120 \mathrm{kVp}$ with tube current modulation (range 30-140 mA). Images were viewed at window settings optimised for the assessment of the lung parenchyma (width 1500 H.U.; level -500 H.U.).

\section{Visual CT analysis}

The CT scoring methodology has been previously described in detail [6] and involved quantifying the following parenchymal pattern extents: ground glass opacity, reticular pattern, honeycombing, emphysema, consolidation and traction bronchiectasis on a lobar basis (Fig. 2). Parenchymal pattern extents were then averaged across six lobes to generate an average lung score for each pattern. Each CT was scored independently by two of four radiologists (AN, SLF, RE, ALB) with 5 to 9 years thoracic imaging experience, blinded to all clinical information and the time points of the serial CTs. For both visual and CALIPER analysis, total fibrosis extent represented the sum of reticulation and honeycombing, whilst total ILD extent additionally summed ground glass opacification.

\section{Fig. 2}

A CT pattern of ground glass with overlaid reticulation is evident in both lower lobes. So called "ground glass with texture" reflects the observation that, as opposed to areas of pure ground glass opacities, which appear smooth, the topology arising from overlaid reticulation is more complex and textured. Associated fibrosis is evident from the localised traction bronchiectasis in the right lower lobe (adjacent to asterisk). Visual scorers categorised such a pattern as reticulation, whilst the computer scored the pattern predominantly as ground glass opacity 


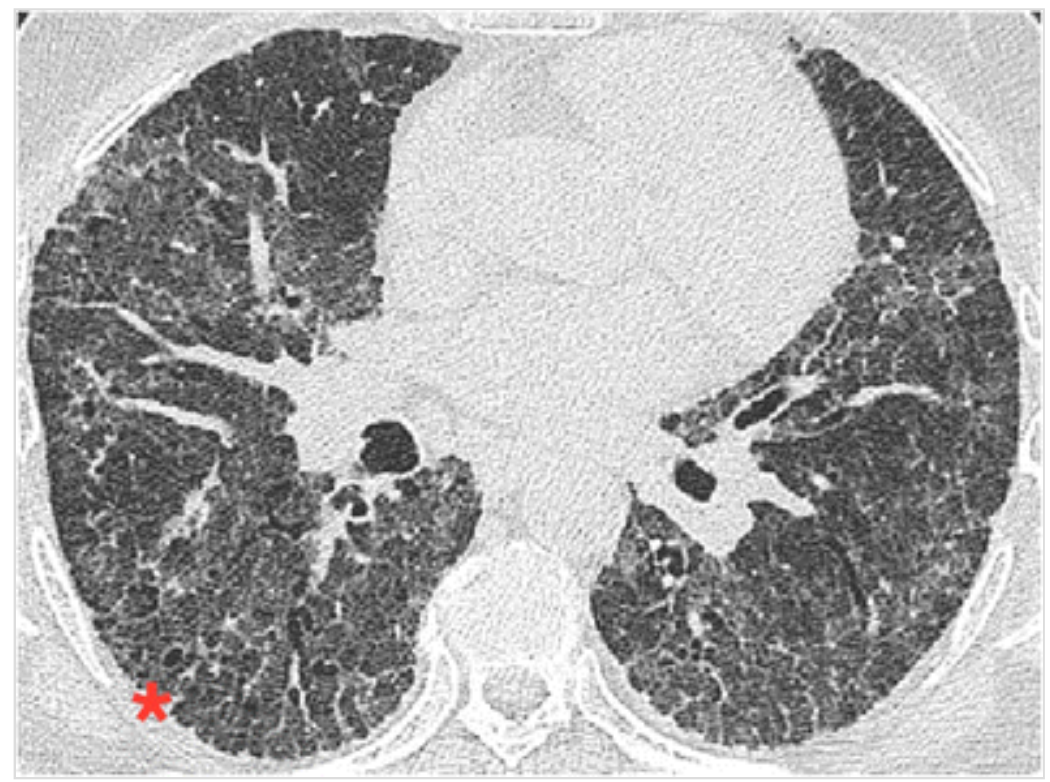

Consensus formulation for visual scores involved identifying systematic biases in visual scores by plotting the spread of differences in parenchymal pattern scores between observers. The most disparate 5\% (two standard deviations) of values were arbitrated by a third scorer for all parameters, thereby minimising bias among the original scorers. If a single parenchymal subtype extent was changed by consensus, the other parameters were modified, following CT review, to retain an overall sum of $100 \%$ for the four parenchymal subtypes. Similarly, if the lobar percentages of total interstitial disease or emphysema varied, the other parameter extent was rescored [7].

Visual lobar CT scores were adjusted prior to statistical analysis to account for the contribution made by each lobe to total lung volume in health. As fibrosis develops, and, in the case of IPF, shrinks the lower lobes disproportionately, the true extent of lung fibrosis can be underestimated when using volumetric scoring systems. The shrunken contracted lower lobes contribute relatively smaller volumes toward the total lung volume than would be the case in health. As a consequence, lower lobes that might have been $25 \%$ of the lung volume in health, when shrunken to $10 \%$ of the total lung volume secondary to fibrosis, cannot reflect the true extent of disease within the lungs when using a volumetric score, as was the case with computer analysis, and traditional visual scoring of 5 axial CT sections.

Lobar scores, as used in the visual scoring of CT scans in the current study, however, more accurately relate the extent of fibrosis within the lung, as each lobe is categorised as a discrete unit, regardless of its post-fibrosis induced volume loss. A potential inaccuracy with visual lobar scoring lies in the relatively high proportional weighting given to the middle lobes, which are each quantified as one sixth of the lung. To account for this, the lobar scores of each individual visual scorer were adjusted to reflect the physiological contribution of 
each lobe to the total lung volume in health (Supplementary Table 1), as characterised using scintigraphic and gas dilution techniques [7]. Therefore the right upper lobe scores were multiplied by $16.4 / 16.7$, where $16.4 \%$ represented the physiological volume of the right upper lobe in health and $16.7 \%$ represented a sixth of the lung volume. The left upper and middle lobes were considered together and adjusted accordingly.

\section{Computer CT analysis}

CALIPER data processing steps have been previously described and involve extraction of the lung from the surrounding thoracic structures and segmentation into upper, middle and lower zones. Lung segmentation was performed with an adaptive density-based morphological approach [8] whilst airway segmentation involved iterative three-dimensional region growing, density thresholding (thresholds including -950HU and -960HU) and connected components analysis. Pulmonary vessels were extracted using optimised multi-scale tubular structure enhancement filters [9]. Parenchymal tissue type classification was applied to $15 \times 15 \times 15$ voxel volume units using texture analysis, computer vision-based image understanding of volumetric histogram signature mapping features and $3 \mathrm{D}$ morphology [10].

Computer evaluation of chest CTs by CALIPER [5] quantified ground glass opacity, reticular pattern, honeycombing and the pulmonary vessel volume (PVV). The PVV represented the total volume of pulmonary arteries and veins, excluding vessels, at the lung hilum. PVV was also subanalysed according to vessel location and vessel size. Using the carina as a landmark, vessels were defined as belonging to the upper zones if they were located above the carina. The distance between the carina and the lowermost CT section containing lung parenchyma was equally divided and used to demarcate the middle and lower zones of the lung. Similarly, vessels were examined according to cross-sectional area thresholds of $<5 \mathrm{~mm}^{2}, 5-10 \mathrm{~mm}^{2}, 10-15 \mathrm{~mm}^{2}, 15-20 \mathrm{~mm}^{2},>20 \mathrm{~mm}^{2}$. CALIPER analysis also quantified three grades of low parenchymal attenuation. Grade 2 and 3 low attenuation areas have previously been shown to correspond to emphysema scored visually, whilst grade 1 low attenuation captured areas that corresponded to lung that appeared morphologically normal on CT [6]. CALIPER did not include quantification of consolidation or traction bronchiectasis.

\section{Calculation of change in $\mathrm{CT}$ and pulmonary function variables}

All CT variables (both visual and computer-derived) were computed as a percentage of the total lung volume except traction bronchiectasis, which was scored using a categorical 4 point scale [7]. Changes in all CT and lung function 
variables were expressed as absolute change per year (by dividing by the time interval between examinations), whilst FVC and DLco change was also expressed as relative change per year using volume measurements, rather than percent predicted values.

\section{Statistical analysis}

Data are given as medians or means with standard deviations, or numbers of patients with percentages where appropriate. Interobserver variation for visual scores was assessed using the single determination standard deviation [11]. Mean population differences between patients with and without emphysema were measured using the Students T-test for continuous variables and the Chi-squared test for categorical variables. Univariate and multivariate linear regression analysis were used to investigate relationships between the three data sets: quantitative CT change, visual CT change and change in forced vital capacity. Statistical analyses were performed with STATA (version 4, StatCorp, College Station, TX, USA).

\section{Results}

\section{Population demographics}

Demographic data, mean visual CT score change, computer score change and pulmonary function test data are provided in Table 1. Interobserver variation values for the visual scores are shown in Supplementary Table 2. The median patient age was 66 years and $80 \%$ of patients were male. The mean interval between CTs and pulmonary function tests was the same at 1.1 years.

\section{Table 1}

Patient age, gender and measures of change per year in various pulmonary function indices, CALIPER and visually scored CT. Data represent mean values with standard deviations unless otherwise indicated. FEV1, FVC, DLco and Kco were measured as relative change. $\mathrm{FEV} 1$ = forced expiratory volume in one second, $\mathrm{FVC}=$ forced vital capacity, DLco $=$ diffusing capacity for carbon monoxide, $\mathrm{CT}=$ computed tomography, $\mathrm{PFT}=$ pulmonary function test, $\mathrm{ILD}=$ interstitial lung disease, $\mathrm{TxBx}=$ traction bronchiectasis, $\mathrm{PVV}=$ pulmonary vessel volume, CALIPER=Computer Aided Lung Informatics for Pathology Evaluation and Rating, Biomedical Imaging Resource, Mayo Clinic Rochester, USA

\section{Variable ( $n=66$ unless stated) \\ Units are percentage unless stated}

\section{Value}

\section{5}

$53 / 13$

Male/female (numbers)

$20 / 46$ 
Variable ( $\mathrm{n}=66$ unless stated)

Units are percentage unless stated

Value

Never/ever smokers (numbers)

$18 / 48$

Mean CT time interval (years)

$1.1 \pm 0.4$

Mean PFT time interval (years)

$1.1 \pm 0.4$

Relative FEV1 change per year

$-8.7 \pm 11.9$

Relative FVC change per year

$-10.4 \pm 16.3$

Relative DLco change per year

$-18.7 \pm 18.3$

CALIPER ILD extent change per year

$7.8 \pm 15.1$

CALIPER Ground glass opacity change per year

$5.3 \pm 12.6$

CALIPER Reticular pattern change per year

$2.3 \pm 4.0$

CALIPER Honeycombing change per year

$0.2 \pm 0.6$

CALIPER Emphysema change per year

$-0.0 \pm 1.6$

CALIPER PVV change per year

$0.9 \pm 1.3$

CALIPER Normal lung change per year

$-8.6 \pm 16.2$

Visual ILD extent change per year

$5.5 \pm 14.5$

Visual Ground glass opacity change per year

$-1.0 \pm 14.4$

Visual Reticular pattern change per year

$4.3 \pm 13.0$

Visual Honeycombing change per year

$2.1 \pm 7.8$

Visual Consolidation change per year

$0.2 \pm 5.1$

Visual Emphysema change per year

$0.9 \pm 3.3$

Visual TxBx severity change per year (max score 18)

$1.4 \pm 3.3$

\section{Morphological and functional longitudinal change}

The amount of normal lung characterised visually and by computer decreased by $7.9 \%$ and $8.6 \%$ per year, respectively, in absolute terms. Computer analysis identified slightly more yearly change in total ILD (7.8\%) than visual scores $(5.5 \%)$. With regard to individual ILD parenchymal patterns, computer-derived ground glass opacity was the pattern that increased the most per year $(5.3 \%)$, followed by reticular pattern $(2.3 \%)$ [Table 1 ]. On visual scoring, however, traction bronchiectasis severity, measured on an 18-point scale, increased by $1.4 \%$ annually whilst reticular pattern and honeycombing increased annually by $4.3 \%$ and $2.1 \%$, respectively (Table 1 ). 
The pulmonary vessel volume (PVV), representing the percentage of the lung that was composed of vessels (computer score only), increased in absolute terms by $0.9 \%$ annually (Table 1, Fig. 3 ). The pulmonary vessels comprised $4.7 \pm 1.6$ percent of the lung on the baseline $\mathrm{CT}$, which increased to $5.6 \pm 2.2$ percent of the lung at the time of the second CT, equating to a relative increase in PVV of $19.1 \%$. When PVV was quantified as a pure volumetric measure, without adjustment for total lung volume and conversion to percentage of the lung, the vessel volume was shown to increase from $176.0 \mathrm{ml}$ to $189.8 \mathrm{ml}$, equating to a relative annual volume change of $7.8 \%$.

\section{Fig. 3}

CALIPER (Computer Aided Lung Informatics for Pathology Evaluation and Rating, Biomedical Imaging Resource, Mayo Clinic Rochester, USA) colour overlay images on two axial CT slices highlighting pulmonary vessels coloured according to size in a 77-year-old never smoker with IPF diagnosed by a multidisciplinary team. The initial CT (a) demonstrates peripheral reticulation and traction bronchiectasis. In a subsequent CT performed just over 15 months later (b), the pulmonary vessel volume has increased from $4.6 \%$ of the lung to $4.9 \%$ of the lung. Interstitial lung disease extent as measured by computer analysis increased from 13.7 to $16.3 \%$ of the lung over the same time interval, though the difference can be hard to discern using visual CT analysis alone. Vessels are colour coded according to size: $<5 \mathrm{~mm}^{2}=$ red, $5-10 \mathrm{~mm}^{2}=$ green, $10-15 \mathrm{~mm}^{2}=$ yellow, 15 $20 \mathrm{~mm}^{2}=$ purple, $>20 \mathrm{~mm}^{2}=$ blue 


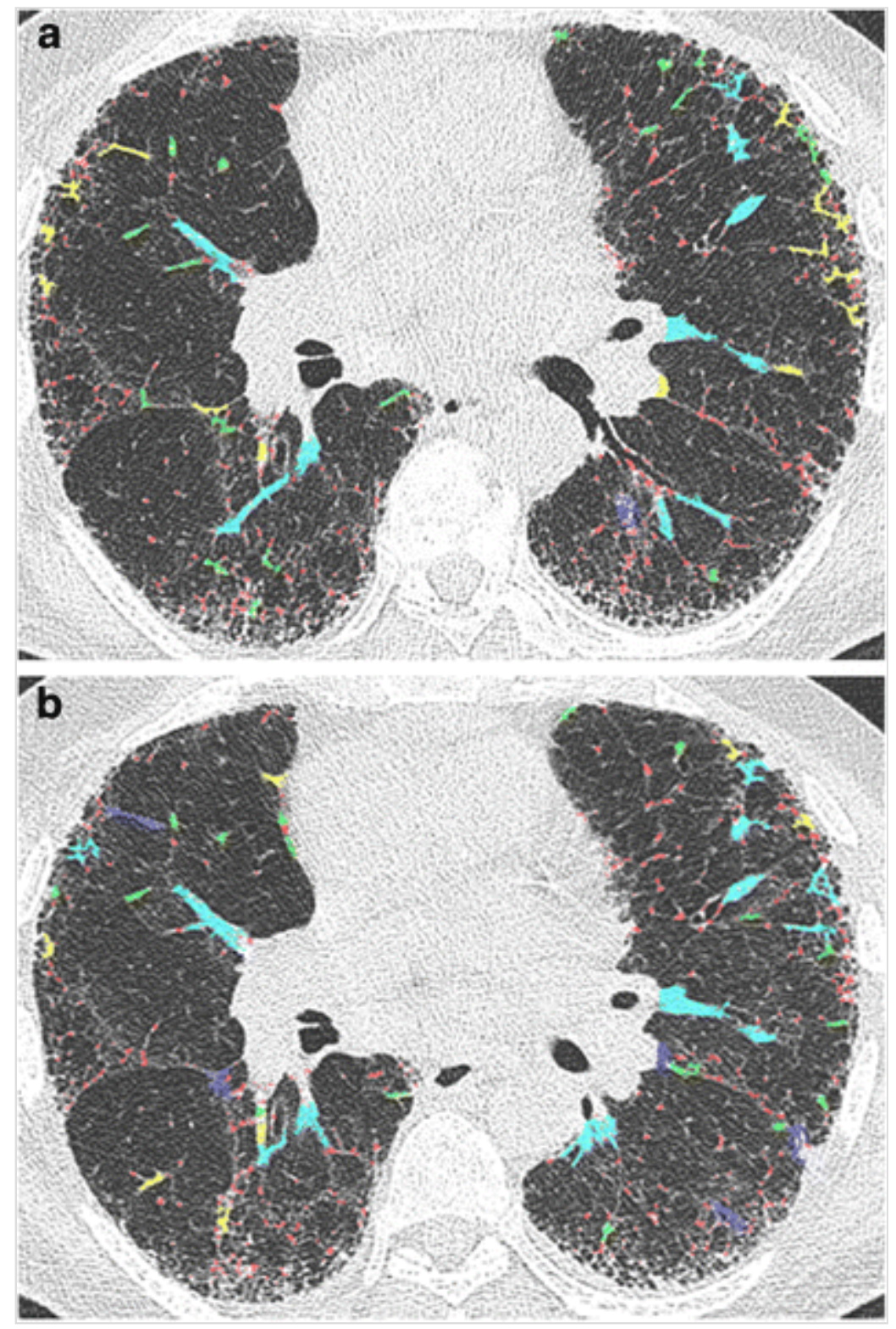

\section{Effects of emphysema presence on change in CT variables}

Whist across the entire population, DLco levels showed a much larger relative annual decrease (18.7\%) than FVC (10.4\%) levels, the differences were exaggerated in patients in whom emphysema was visually identified on the baseline CT (Table 2). Annualised relative DLco declines were similar in patients with (19.6\%) and without emphysema (17.4\%). However, annual relative FVC declines were markedly attenuated in patients with emphysema, with the differences showing a trend towards statistical significance $(p=0.09)$. In patients with emphysema, a significantly smaller increase in traction bronchiectasis severity was noted across the two scans when compared to patients without emphysema (Table 2).

\section{Table 2}

Variation in longitudinal measures of pulmonary function indices and CALIPER and visually scored CT variables according to the presence or absence of emphysema. Change in all variables was standardised as change per year. Data represent mean values with 
standard deviations. Differences between groups were measured using the Students T-test for continuous variables and the Chi-squared test for categorical variables. ${ }^{*}=$ significant differences to a significance level of $\mathrm{p}<0.05$. FEV1, FVC and DLco were measured as relative change. FEV1 = forced expiratory volume in one second, FVC = forced vital capacity, DLco = diffusing capacity for carbon monoxide, ILD=interstitial lung disease, $\mathrm{TxBx}=$ traction bronchiectasis, $\mathrm{PVV}=$ pulmonary vessel volume, CALIPER=Computer Aided Lung Informatics for Pathology Evaluation and Rating, Biomedical Imaging Resource, Mayo Clinic Rochester, USA

\begin{tabular}{|c|c|c|}
\hline $\begin{array}{l}\text { Variable }(n=66 \text { unless stated }) \\
\text { Units are percentage unless stated }\end{array}$ & No emphysema $(n=37)$ & Emphysema $(n=29)$ \\
\hline Median Age (years) & 65 & 69 \\
\hline Male/female & $30 / 7$ & $23 / 6$ \\
\hline Never/ever smokers & $13 / 24$ & $5 / 24$ \\
\hline Alive/dead & $13 / 24$ & $7 / 22$ \\
\hline Mean follow up time (months) & $21.4 \pm 16.4$ & $23.1 \pm 15.7$ \\
\hline Mean CT time interval (years) & $1.1 \pm 0.36$ & $1.2 \pm 0.37$ \\
\hline Mean PFT time interval (years) & $1.1 \pm 0.36$ & $1.1 \pm 0.37$ \\
\hline FEV1 & $-11.3 \pm 12.5$ & $-5.5 \pm 10.5^{*}$ \\
\hline FVC & $-13.4 \pm 17.7$ & $-6.7 \pm 13.6$ \\
\hline DLco & $-19.6 \pm 20.3$ & $-17.4 \pm 15.7$ \\
\hline CALIPER ILD extent & $7.6 \pm 16.3$ & $8.0 \pm 13.6$ \\
\hline CALIPER Ground glass opacity & $4.9 \pm 14.2$ & $5.7 \pm 10.4$ \\
\hline CALIPER Reticular pattern & $2.4 \pm 4.1$ & $2.2 \pm 3.8$ \\
\hline CALIPER Honeycombing & $0.2 \pm 0.6$ & $0.1 \pm 0.6$ \\
\hline CALIPER Emphysema & $-0.1 \pm 1.8$ & $-0.0 \pm 1.5$ \\
\hline CALIPER PVV & $1.0 \pm 1.4$ & $0.8 \pm 1.3$ \\
\hline CALIPER Normal lung & $-8.5 \pm 17.6$ & $-8.7 \pm 14.5$ \\
\hline Visual ILD extent & $5.2 \pm 13.8$ & $6.0 \pm 15.5$ \\
\hline Visual Ground glass opacity & $-3.0 \pm 17.9$ & $1.6 \pm 7.6$ \\
\hline Visual Reticular pattern & $4.1 \pm 13.8$ & $4.6 \pm 12.2$ \\
\hline Visual Honeycombing & $3.0 \pm 7.0$ & $0.9 \pm 8.7$ \\
\hline Visual Consolidation & $0.4 \pm 6.6$ & $-0.0 \pm 2.1$ \\
\hline Visual Mosaic attenuation & $0.6 \pm 3.0$ & $0.4 \pm 1.7$ \\
\hline Visual Emphysema & $0.0 \pm 0.0$ & $1.8 \pm 4.8^{*}$ \\
\hline
\end{tabular}


Variable ( $n=66$ unless stated)

Units are percentage unless stated

Visual TxBx severity (max score 18 )
No emphysema $(n=37)$

Emphysema $(n=29)$

$2.1 \pm 3.7$

$0.4 \pm 2.5^{*}$

\section{Regression analyses}

Univariate linear regression analysis was performed for all CT patterns to identify associations with FVC change (Table 3). Visual CT parameters were less strongly linked to FVC change than computer-derived parameters, as measured by $\mathrm{R}^{2}$ values (Table 3 ). Change in traction bronchiectasis severity was found to be the strongest visual predictor of $F V C$ change $\left(\mathrm{R}^{2}=0.40\right)$.

\begin{tabular}{llll}
\hline $\begin{array}{l}\text { Pulmonary } \\
\begin{array}{l}\text { Function Test } \\
\text { change/yr }\end{array}\end{array}$ & CT pattern & Beta & $95 \%$ Confidence P v \\
change/yr & Coefficient & Interval
\end{tabular}

\begin{tabular}{lllll}
\hline Forced vital & Total ILD extent & -0.45 & $-0.71,-0.19$ & $0 . C$
\end{tabular}


Ground glass opacity 0.32

$0.05,0.59$

Reticular pattern

$-0.63$

$-0.90,-0.36$

$<0$.

Honeycombing

$-0.21$

$-0.73,0.31$

0.

Total emphysema

0.76

$-0.46,1.98$

0.

Consolidation

$-0.83$

$-1.60,-0.06$

0.1

TxBx severity

$-3.06$

$-4.01,-2.12$

$<0$.

\section{Forced vita \\ capacity}

Total ILD extent

$-0.78$

$-0.97,-0.60$

$<0$.

$\begin{array}{lll}\text { Ground glass opacity-0.85 } & -1.10,-0.61 & <0 . \\ \text { Reticular pattern } \quad-2.65 & -3.43,-1.86 & <0 .\end{array}$

Honeycombing $\quad-3.68 \quad-10.35,2.98 \quad 0.4$

$\begin{array}{llll}\text { Emphysema } & 0.96 & -1.51,3.43 & 0 .\end{array}$

PVV $\quad-9.24 \quad-11.25,-7.23<0$.

Normal Lung

0.73

$0.56,0.90$

$<0$.

\section{Table 3}

Univariate analysis of annual change in visual (white) and CALIPER (light grey) CT variables predictive of yearly change in forced vital capacity. ILD=interstitial lung disease, $\mathrm{TxBx}=$ traction bronchiectasis, $\mathrm{PVV}=$ pulmonary vessel volume, CALIPER=Computer Aided Lung Informatics for Pathology Evaluation and Rating, Biomedical Imaging Resource, Mayo Clinic Rochester, USA

Of all the visual and computer-derived measures of change on $\mathrm{CT}$, change in PVV linked most strongly to FVC change $\left(\mathrm{R}^{2}=0.57\right)$. When various expressions of PVV change were examined (change in absolute and relative percentage PVV and relative volumetric PVV change), absolute PVV change expressed as a percentage of the lung demonstrated the strongest relationship with relative FVC change. Accordingly, PVV change has henceforth been analysed as absolute PVV change. Measures of interstitial fibrosis, such as total ILD extent and reticular pattern, had significant FVC correlations using visual and computerderived scoring methods, but linkages were weaker using visual scores $\left(\mathrm{R}^{2}=0.16\right.$ 
and 0.25 respectively) than when scored by computer $\left(\mathrm{R}^{2}=0.53\right.$ and 0.42 respectively)[Table 3].

Multivariate linear regression analyses were performed to first identify visual and computer-derived parameters independently associated with FVC change in separate models (Table 4). An increase in traction bronchiectasis was the strongest visual variable linked to FVC decline, whilst an increase in PVV was the strongest computer-derived variable linked to FVC decline. Overall, computer-derived variables were found to better explain declines in FVC when compared to visual CT scores (Table 4).

\begin{tabular}{lllll}
\hline $\begin{array}{l}\text { Pulmonary } \\
\text { function } \\
\text { test }\end{array}$ & CT Pattern & Beta & $\begin{array}{l}95 \% \\
\text { Confidence }\end{array}$ & P valu \\
\hline FVC & ILD extent & -0.23 & $-0.45,-0.01$ & 0.04 \\
& Consolidation & -0.84 & $-1.43,-0.26$ & 0.005 \\
& TxBx severity & -2.63 & $-3.59,-1.67$ & $<0.006$ \\
& Ground glass opacity & -0.50 & $-0.84,-0.16$ & 0.004 \\
& Honeycombing & -6.65 & $-11.29,-2.01$ & 0.006 \\
& PVV & -5.66 & $-8.71,-2.60$ & $<0.006$ \\
& TxBx severity & -1.43 & $-2.34,-0.52$ & 0.003 \\
& PVV & -7.17 & $-9.47,-4.86$ & $<0.006$ \\
\hline
\end{tabular}

\section{Table 4}

Combined multivariate linear regression demonstrating relationships significant to a level of 0.05 between parenchymal patterns characterised by visual (white) and CALIPER (light grey) scores and combined visual and CALIPER scores (dark grey) with pulmonary function indices. ILD=interstitial lung disease, $\mathrm{TxBx}=$ traction bronchiectasis, $\mathrm{PVV}=$ pulmonary vessel volume, $\mathrm{FVC}=$ forced vital capacity, $\mathrm{CALIPER}=\mathrm{Computer}$ Aided Lung Informatics for Pathology Evaluation and Rating, Biomedical Imaging Resource, Mayo Clinic Rochester, USA

\section{Subanalysis of PVV change}


The pulmonary vessels were sub-categorised in terms of vessel location and size, and relationships between the various subdivisions of vessels and FVC change were evaluated (Table 5). On univariate analysis of vessel location, vessels in the middle zones of the lung had the strongest linkage $\left(\mathrm{R}^{2}=0.57\right)$ with $\mathrm{FVC}$ change, with results maintained on multivariate analysis. The strength of linkage between middle zone vessels and FVC change was similar in magnitude to univariate relationships between total PVV change and FVC change $\left(\mathrm{R}^{2}=0.57\right)$.

\section{Table 5}

Univariate (white) linear regression analyses demonstrating relationships significant to a level of 0.05 between change in pulmonary vessel volume (PVV) location (top) and pulmonary vessel volume (PVV) size (bottom) and relative change in forced vital capacity (FVC)

\begin{tabular}{|c|c|c|c|c|c|}
\hline $\begin{array}{c}\text { Pulmonary } \\
\text { function test }\end{array}$ & $\begin{array}{c}\text { Vessel } \\
\text { subdivision }\end{array}$ & $\begin{array}{l}\text { Beta } \\
\text { Coeff }\end{array}$ & $\begin{array}{l}95 \% \text { Confidence } \\
\text { Interval }\end{array}$ & P value & $\begin{array}{c}\text { Model } \\
\mathbf{R}^{2}\end{array}$ \\
\hline \multirow{3}{*}{$\begin{array}{l}\text { Relative FVC } \\
\text { change }\end{array}$} & $\begin{array}{l}\text { Upper zone } \\
\text { PVV }\end{array}$ & -16.91 & $-24.23,-9.59$ & 0.0001 & 0.25 \\
\hline & $\begin{array}{l}\text { Middle zone } \\
\text { PVV }\end{array}$ & -20.77 & $-25.31,-16.23$ & $<0.0001$ & 0.57 \\
\hline & $\begin{array}{l}\text { Lower zone } \\
\text { PVV }\end{array}$ & -20.34 & $-27.06,-13.62$ & $<0.0001$ & 0.36 \\
\hline \multirow{5}{*}{$\begin{array}{l}\text { Relative FVC } \\
\text { change }\end{array}$} & $\mathrm{PVV}<5 \mathrm{~mm}^{2}$ & -25.96 & $-33.96,-17.97$ & $<0.0001$ & 0.40 \\
\hline & $\begin{array}{l}\text { PVV 5- } \\
10 \mathrm{~mm}^{2}\end{array}$ & -68.76 & $-89.65,-47.86$ & $<0.0001$ & 0.40 \\
\hline & $\begin{array}{l}\text { PVV 10- } \\
15 \mathrm{~mm}^{2}\end{array}$ & -78.26 & $-107.51,-49.02$ & $<0.0001$ & 0.31 \\
\hline & $\begin{array}{l}\text { PVV 15- } \\
20 \mathrm{~mm}^{2}\end{array}$ & -127.55 & $-163.43,-91.68$ & $<0.0001$ & 0.44 \\
\hline & $\begin{array}{l}\mathrm{PVV} \\
>20 \mathrm{~mm}^{2}\end{array}$ & -14.72 & $-18.68,-10.75$ & $<0.0001$ & 0.46 \\
\hline
\end{tabular}

When vessels of differing size were explored in univariate analyses, all five subdivisions of vessel size had similar linkages with FVC change as judged by $\mathrm{R}^{2}$ values (Table 5). When each vessel size subdivision was examined in a bivariate model with total PVV change, total PVV change retained significance in every model, but only vessels between $10-15 \mathrm{~mm}^{2}$ remained weakly independently associated with FVC change $(p=0.02)$.

Given the strength of the association between middle zone vessels and FVC change, a multivariate model incorporating change in vessel size subdivisions 
and middle zone vessels was examined; this demonstrated change in middle zone vessels to have the strongest independent association with FVC change $(p<0.0001)$. The association between change in middle zone vessels and FVC change was maintained regardless of the presence $\left(\mathrm{R}^{2}=0.42, \mathrm{p}<0.0001\right)$ or absence $\left(\mathrm{R}^{2}=0.63, \mathrm{p}<0.0001\right)$ of emphysema. Following correction for baseline disease severity using either visual fibrosis extent, FVC or DLco in separate models, middle zone vessel change consistently maintained a strong independent link with FVC change ( $p<0.0001$ in each model) in contrast to the variables representative of baseline disease severity.

\section{Discussion}

The current study has identified for the first time that in the longitudinal analysis of patients with IPF, an increase in a computer-derived variable, the pulmonary vessel volume, is the $\mathrm{CT}$ variable most strongly association with a decline in FVC, which itself is the current best measure of a clinical deterioration in a patient with IPF. Specifically, an increase in vessels in the mid-zones of the lungs demonstrated the strongest correlations with measures of functional decline.

Only one previous study, by Lee at al [3], has evaluated change in CT parameters against change in pulmonary function indices. The study by Lee et al [3] evaluated patients with either histologically proven UIP or NSIP and demonstrated similar correlations between change in ILD extent and FVC change in both UIP and NSIP groups ( $\mathrm{R}^{2}=0.20$ and $\mathrm{R}^{2}=0.29$ respectively) [3]. Results were similar to the equivalent visual correlations in the current study, but weaker than the correlations shown between computer-derived ILD extent and FVC change.

FVC decline is the parameter most widely used to evaluate disease progression in IPF $[12,13,14]$ and whilst change in computer-derived variables were a stronger predictor of FVC change than change in visual scores, PVV change was the strongest single variable in the combined multivariate model. PVV increased by $0.9 \%$ of the lung in absolute terms, which reflected a relative increase of $19.1 \%$ by the time of the second CT. Absolute change of a magnitude of $0.9 \%$ of the lung would not be readily discernible to the human eye, and might explain the relative neglect of PVV as a marker of IPF progression to date. Furthermore, the observation that the actual volume of pulmonary vessels increased over time refutes the possibility that an overall reduction in total lung volume, consequent to the fibrotic process, could result in a relative preservation of vascular volume, explaining the increase in PVV over serial CTs. 
Declines in FVC were most strongly related to an increase in vessel volume in the lung mid-zones, rather than change in vessel size. The findings suggest that firstly, any potential misclassification of reticulation as pulmonary vessels (which would be expected to be incorporated in the PVV $<5 \mathrm{~mm}^{2}$ category) has a negligible functional effect when compared to a zonal increase in vessel volumes. Secondly, worsening pulmonary hypertension would be expected to increase vessel size rather than overall zonal vessel volumes, suggesting that our findings do not simply reflect an increase in pulmonary pressures. In addition, computer-derived quantitation of PVV excludes the large vessels at the lung hilum, therefore limiting the possibility that the increase in middle zone vessels reflects enlargement of the main pulmonary arteries. Importantly, regardless of the measure used to characterise baseline disease severity in IPF, an increase in the vessels in the lung mid-zone maintained strong independent functional associations.

Discordances between visual and computer-derived CT scores for change in individual ILD pattern extents were widest for change in ground glass opacity. The finding can partly be explained by a pattern that can be termed "ground glass with texture", whereby a pattern of fine reticulation lies overlaid on ground glass opacities (Fig. 2). Such a pattern was generally preferentially scored as ground glass opacity by computer analysis, and more often scored visually as reticulation, reflecting biases both from the radiologists visually scoring the CTs and the radiologists who initially trained the computer tool and derived its exemplars for ground glass opacity and reticular pattern.

Differences in extent of honeycomb change between visual and computer-derived scores may be multifactorial. A degree of misclassification of cystic disease occurs with computer analysis with cyst walls labelled as reticular pattern, and cysts themselves labelled as emphysema or even normal lung. More importantly, as previously described, and analogous to baseline comparisons of visual and computer-derived scores [6], longitudinal change of ILD extent and its components are under-represented by computer analysis when compared to visual scores as a consequence of differences in CT scoring methodology. As fibrosis extent increases over time, more of the damaged lung volume contracts, and compensatory hyper-expansion of normal lung follows (the upper and middle lobes in IPF). Consequently, whilst the extent of fibrosis may increase in absolute terms, this may translate to a relatively smaller volume of lung considered to be fibrotic. A volumetric CT score (as performed using computer analysis), will therefore underestimate a longitudinal increase in reticular pattern and honeycombing extents when compared to visual scores which, when 
performed on a lobar basis, ascribe equal weight to the contracted lower lobes and the hyper-expanded upper lobes.

Several previous studies have confirmed the relative preservation of lung volumes that occurs secondary to the presence of emphysema in patients with IPF $[15,16,17]$. Our findings demonstrated that FVC decline (but not DLco decline) was attenuated in the presence of emphysema, though the relatively small patient numbers limit interpretation of the observation. However, given that FVC declines are both the primary clinical measure of deterioration in IPF [18] and represent an established drug trial end-point [1], it is possible that the purported $30-40 \%$ of IPF patients that have emphysema [17, 19, 20, 21] may have important functional declines that are unrecognised. Our observations have importance both clinically and for drug trials, but will require validation and correlation with patient outcomes in larger prospective IPF cohorts.

An increase in traction bronchiectasis severity was the visual CT parameter that linked most strongly with FVC decline. Our findings are in line with previous work demonstrating linkages between traction bronchiectasis and mortality [22, $23,24,25]$, traction bronchiectasis and fibroblastic foci profusion [26, 27] and between fibroblastic foci profusion and outcome in IPF [28, 29, 30].

Interestingly, in IPF patients with emphysema, traction bronchiectasis worsened at a significantly slower rate than in patients without emphysema. As previously observed [31], it is conceivable that pre-existing emphysema-induced damage to the connective tissue scaffold of the lung may limit the degree to which airways can be distracted by supervening interstitial fibrosis when compared to nonfibrotic lung. Accordingly, over time, traction bronchiectasis may also progress at a reduced rate when emphysema co-exists with fibrosis.

There were limitations to the current study. Firstly, the pulmonary function tests were performed within 3 months of the corresponding CT scan. Thus, when serial CT scans were compared, the time interval between $\mathrm{CT}$ imaging and pulmonary function testing may have been different. In practice, however, all change scores were normalised to a yearly change. Furthermore, when the mean and standard deviations of CT and pulmonary function test intervals were calculated, the results were similar (Table 1). With regard to CT intervals assessed in the current study, ideally a narrower time frame such as 9-18 months would have been used to replicate the optimal clinical window within which a patient might routinely be re-scanned to assess disease progression. A follow-up period of 6-24 months was chosen for analysis, as it provided a great number of patients from which to analyse structure-function relationships. 
In conclusion, the current study has demonstrated that an increase in an automated computer-derived variable, the pulmonary vessel volume, is the strongest CT determinant of FVC decline in IPF. Specifically, an increase in vessels in the middle zones of the lung demonstrated the strongest linkages with functional deterioration and was independent of measures of baseline disease severity. An increase in traction bronchiectasis severity was the visual CT score that demonstrated the strongest linkages with functional decline.

\section{Funding}

The authors state that this work has not received any funding.

Compliance with ethical standards

Guarantor The scientific guarantor of this publication is Prof Athol Wells.

Conflict of interest The authors of this manuscript declare relationships with the following companies:

Dr. Jacob reports personal fees from Boehringer Ingelheim, outside the submitted work.

BJB, RK, SR report a grant from the Royal Brompton Hospital during the conduct of the study; another from Imbio, LLC, was outside the submitted work; and all have a patent: SYSTEMS AND METHODS FOR ANALYZING IN VIVO TISSUE VOLUMES USING MEDICAL IMAGING DATA licensed to Imbio, LLC.

Dr. Wells reports personal fees from Intermune, personal fees from Boehringer Ingelheim, personal fees from Gilead, personal fees from MSD, personal fees from Roche, personal fees from Bayer, personal fees from Chiesi, outside the submitted work.

Dr. Walsh reports personal fees from Boehringer Ingelheim, personal fees from Roche, outside the submitted work.

The manuscript was supported by the National Institute of Health Research Respiratory Disease Biomedical Research Unit at the Royal Brompton and Harefield NHS Foundation Trust and Imperial College London.

Statistics and biometry One of the authors has significant statistical expertise. 
Informed consent Written informed consent was waived by the Institutional Review Board.

Ethical approval Institutional Review Board approval was obtained.

Study subjects or cohorts overlap Some study subjects or cohorts have been previously reported in two studies: a structure function study and a mortality study. However these examined CT and functional variables at baseline and did not look at longitudinal change.

Methodology $\cdot$ retrospective

- observational

- performed at one institution

\section{Electronic supplementary material}

\section{$\mathrm{AQ3}$}

\section{ESM 1}

(DOCX $60 \mathrm{~kb})$

\section{References}

\section{$\mathrm{AQ}_{4}$}

1. Raghu G, Collard HR, Egan JJ et al (2011) An official

ATS/ERS/JRS/ALAT statement: idiopathic pulmonary fibrosis-evidencebased guidelines for diagnosis and management. Am J Respir Crit Care Med 183:788-824

2. Jacob J, Bartholmai B, Rajagopalan S et al (2016) Mortality prediction in idiopathic pulmonary fibrosis: evaluation of automated computer tomographic analysis with conventional severity measures. Eur Respir J. https://doi.org/10.1183/13993003.01011-2016

3. Lee HY, Lee KS, Jeong YJ et al (2012) High-Resolution CT Findings in Fibrotic Idiopathic Interstitial Pneumonias With Little Honeycombing: Serial Changes and Prognostic Implications. Am J Roentgenol 199:982-989

4. Nagao T, Nagai S, Hiramoto Y et al (2002) Serial evaluation of highresolution computed tomography findings in patients with idiopathic 
pulmonary fibrosis in usual interstitial pneumonia. Respiration 69:413-419

5. Maldonado F, Moua T, Rajagopalan S et al (2014) Automated quantification of radiological patterns predicts survival in idiopathic pulmonary fibrosis. Eur Respir J 43:204-212

6. Jacob J, Bartholmai B, Rajagopalan S et al (2016) Automated quantitative $\mathrm{CT}$ versus visual $\mathrm{CT}$ scoring in idiopathic pulmonary fibrosis: validation against pulmonary function. J Thorac Imaging 31:304-311

7. Pierce RJ, Brown DJ, Denison DM (1980) Radiographic, scintigraphic, and gas-dilution estimates of individual lung and lobar volumes in man. Thorax 35:773-780

8. Hu S, Hoffman EA, Reinhardt JM (2001) Automatic lung segmentation for accurate quantitation of volumetric X-ray CT images. IEEE Trans Med Imaging 20:490-498

9. Shikata H, McLennan G, Hoffman EA, Sonka M (2009) Segmentation of pulmonary vascular trees from thoracic 3D CT images. Int J Biomed Imaging. 11

AQ5

10. Bartholmai BJ, Raghunath S, Karwoski RA et al (2013) Quantitative CT imaging of interstitial lung diseases. J Thorac Imaging 28:298-307

11. Chinn S (1991) Repeatability and method comparison. Thorax 46:454456

12. Flaherty KR, Mumford JA, Murray S et al (2003) Prognostic implications of physiologic and radiographic changes in idiopathic interstitial pneumonia. Am J Respir Crit Care Med 168:543-548

13. Collard HR, King TE Jr, Bartelson BB et al (2003) Changes in clinical and physiologic variables predict survival in idiopathic pulmonary fibrosis. Am J Respir Crit Care Med 168:538-542

14. du Bois RM, Weycker D, Albera C et al (2011) Forced Vital Capacity in Patients with Idiopathic Pulmonary Fibrosis. Am J Respir Crit Care Med 184:1382-1389 
15. Mura M, Zompatori M, Pacilli AM et al (2006) The presence of emphysema further impairs physiologic function in patients with idiopathic pulmonary fibrosis. Respir Care 51:257-265

16. Wiggins J, Strickland B, Turner-Warwick M (1990) Combined cryptogenic fibrosing alveolitis and emphysema: the value of high resolution computed tomography in assessment. Respir Med 84:365-369

17. Ryerson C, Hartman T, Elicker B et al (2013) Clinical features and outcomes in combined pulmonary fibrosis and emphysema in idiopathic pulmonary fibrosis. Chest 144:234-240

18. King TE, Safrin S, Starko KM et al (2005) Analyses of efficacy end points in a controlled trial of interferon-gamma $1 \mathrm{~b}$ for idiopathic pulmonary fibrosis. Chest 127:171-177

19. Wells AU, King AD, Rubens MB et al (1997) Lone cryptogenic fibrosing alveolitis: a functional-morphologic correlation based on extent of disease on thin-section computed tomography. Am J Respir Crit Care Med 155:13671375

20. Wells AU, Desai SR, Rubens MB et al (2003) Idiopathic pulmonary fibrosis: a composite physiologic index derived from disease extent observed by computed tomography. Am J Respir Crit Care Med 167:962-969

21. Mitchell PD, Das JP, Murphy DJ et al (2015) Idiopathic Pulmonary Fibrosis With Emphysema: Evidence of Synergy Among Emphysema and Idiopathic Pulmonary Fibrosis in Smokers. Respir Care 60:259-268

22. Edey AJ, Devaraj AA, Barker RP et al (2011) Fibrotic idiopathic interstitial pneumonias: HRCT findings that predict mortality. Eur Radiol $21: 1586-1593$

23. Sumikawa H, Johkoh T, Colby TV et al (2008) Computed tomography findings in pathological usual interstitial pneumonia: relationship to survival. Am J Respir Crit Care Med 177:433-439

24. Walsh SL, Sverzellati N, Devaraj A et al (2012) Chronic hypersensitivity pneumonitis: high resolution computed tomography patterns and pulmonary function indices as prognostic determinants. Eur Radiol 22:1672-1679 
25. Walsh SL, Sverzellati N, Devaraj A et al (2014) Connective tissue disease related fibrotic lung disease: high resolution computed tomographic and pulmonary function indices as prognostic determinants. Thorax 69:216-222

26. Walsh SLF, Wells AU, Sverzellati N et al (2015) Relationship between fibroblastic foci profusion and high resolution $\mathrm{CT}$ morphology in fibrotic lung disease. BMC Med 13:241

27. Chiba S, Tsuchiya K, Akashi T et al (2016) Chronic Hypersensitivity Pneumonitis With a Usual Interstitial Pneumonia-Like Pattern: Correlation Between Histopathologic and Clinical Findings. Chest 149:1473-1481

28. King TE Jr, Schwarz MI, Brown K et al (2001) Idiopathic pulmonary fibrosis: relationship between histopathologic features and mortality. Am J Respir Crit Care Med 164:1025-1032

29. Nicholson AG, Fulford LG, Colby TV et al (2002) The relationship between individual histologic features and disease progression in idiopathic pulmonary fibrosis. Am J Respir Crit Care Med 166:173-177

30. Harada T, Watanabe K, Nabeshima K et al (2013) Prognostic significance of fibroblastic foci in usual interstitial pneumonia and non-specific interstitial pneumonia. Respirology 18:278-283

31. Desai SR, Wells AU, Rubens MB et al (2003) Traction bronchiectasis in cryptogenic fibrosing alveolitis: associated computed tomographic features and physiological significance. Eur Radiol 13:1801-1808 